\title{
Heterosis in two closely related species of earthworm (Eisenia fetida and $E$. andrei)
}

\author{
THOMAS C. McELROY $\dagger^{*} \&$ WALTER J. DIEHL $\ddagger$ \\ $\uparrow$ Department of Biological Sciences, Florida International University, Miami, FL 33199, $₫$ Department of Biological \\ Sciences, Mississippi State University, P.O. Drawer GY, Mississippi State, MS 39762 USA
}

\begin{abstract}
The importance of heterosis, and in particular allozyme-associated heterosis, in natural populations remains unclear. Much of the scepticism that surrounds allozyme-associated heterosis comes from inconsistent and unreliable detection of the relationship. Thus, evaluating the genetic mechanisms that may cause allozyme-associated heterosis in natural populations has proven difficult. The most prevalent hypotheses that have been put forward to explain the genetic basis of heterosis are the general dominance and the local overdominance hypotheses. A factorial crossing design was used to survey eight polymorphic allozyme loci in the parent and offspring generations of two species of earthworms in order to evaluate possible mechanisms of allozyme-associated heterosis for growth rate. Significant heritable variation for growth rate was detected only within a single cross. Allozymeassociated heterosis for growth rate was detected only within this cross. This relationship did not persist after the effects of interfamily variation were removed. These results indicate that simple heritability of a fitness-related trait may be necessary for predictive power and repeatability of allozyme-associated heterosis, and that the allozyme-associated heterosis detected in this study was the result of general dominant genetic effects.
\end{abstract}

Keywords: allozymes, earthworms, Eisenia, growth, heterosis, heterozygosity.

\section{Introduction}

Many hypotheses have been suggested to explain the genetic basis of heterosis (hybrid vigour). The two most prevalent hypotheses are general dominance and local overdominance (Mitton, 1997; David, 1998). The general dominance hypothesis invokes the suppression of deleterious recessive alleles in the background genome from one parent by dominant alleles from the other parent (Bruce, 1910). The local overdominance hypothesis assumes that the heterozygote at a locus is superior in performance to either of the homozygous combinations of alleles (East, 1936). Heterosis may be the result of these or other mechanisms, and there may not be a unifying mechanism among species or even among individuals of a species for heterosis (Hayes, 1952; McElroy et al., 1999).

It is difficult to distinguish general dominance from local overdominance because the hypotheses make overlapping predictions about the effect of heterozygosity on fitness of an organism (Crow, 1952). General dominance heterotic effects are associative overdominant effects that can arise from variance in inbreeding.

*Correspondence. E-mail: mcearthworm@aol.com
General associative overdominance is indicated when resolved loci are marking genomic heterozygosity and they are correlated with loci that are segregating for deleterious recessive alleles (David, 1998). Local overdominance heterotic effects are detected when a resolved locus is marking a chromosomal segment that is functionally overdominant. The functional overdominance may be the result of the marker locus itself (true overdominance) or the marker locus can appear as an overdominant locus if the locus includes tightly linked deleterious recessive alleles in repulsion or coupling phase equilibrium (associative overdominance) (Houle, 1989; Lynch \& Walsh, 1998).

In natural populations, heterosis has often been detected at protein-encoding loci as a positive relationship between multilocus heterozygosity (MLH, number of heterozygous loci out of a set of polymorphic loci) and individual fitness (Mitton, 1997). These relationships have not always been found when expected (McAndrew et al., 1986; Booth et al., 1990; Whitlock, 1993; Mulvey et al., 1994), stimulating much speculation on the adaptive significance of allozyme heterozygosity and allozyme-associated heterosis. When allozymeassociated heterosis has been detected, it is unclear whether the observed relationship was the result of the 
general associative overdominance hypothesis or the local true/associative overdominance hypothesis (Mitton, 1997; David, 1998).

Although allozyme variation occurs in virtually all natural populations studied, a positive correlation between multilocus allozyme heterozygosity and fitness (growth) is not universal (Hartl \& Clark, 1989; Britten, 1996). For example, Pogson \& Zouros (1994) compared allozyme heterozygosity-fitness relationships and noncoding DNA heterozygosity-fitness relationships in scallops (Placopecten magellanicus). Their results suggested that the observed MLH-fitness relationships were restricted to the allozyme markers. These data supported the local overdominance hypothesis for the allozyme loci surveyed. However, Pogson \& Fevolden (1998) attempted a similar comparison in Atlantic cod (Gadus morhua) and found a significant relationship between noncoding DNA heterozygosity and fitness. These data supported the general associative overdominance hypotheses. Thus, evaluation of universal mechanisms that may cause allozyme-associated heterosis has proven difficult.

The experiment reported herein used four populations assumed to have different associations between allozyme markers and deleterious recessive mutations that have occurred in their respective genomes. Fitness of individuals was estimated from early growth rate which has been shown to be correlated with fecundity in one of the species used in this experiment (Eisenia andrei) (Presley et al., 1996). Furthermore, this experiment was performed in a single, controlled environment in order to reduce the confounding effects of environmental variation.

As no consistent patterns of heterozygosity-fitness relationships were found in a series of studies on a population of the earthworm Eisenia andrei (Diehl, 1988; Audo \& Diehl, 1995; McElroy et al., 1997), the present study employed a total of four populations of two closely related species of the earthworm genus Eisenia (E. fetida and E. andrei). A modified factorial mating design with both intra- and interspecific crosses was used to partition the effect of population, family, individual multilocus heterozygosity and single locus heterozygosity on growth rate at different levels of outbreeding in the $\mathrm{F}_{1}$ generation ( $\mathrm{Li} \& \mathrm{Wu}, 1996$ ). Unlike other research, there was no reliance on advanced generations, initiated with inbred lines, in order to preserve the genetic variation that would be present in the natural populations.

Two hypotheses were tested:

1 There is a correlation between increased population heterozygosity and the average heterosis for growth rate of individuals in that population. In order to support this hypothesis the data must show a general relation- ship between allozyme marker heterozygosity and heterosis for growth rate.

2 Allozyme loci are marking local chromosomal segments that contribute directly to heterosis by true/ associative overdominance and are not simply neutral loci marking genomic heterozygosity. In order to support this hypothesis the data must show significant heritable variation for growth rate, thus revealing a potential for selective pressure to favour allozyme heterozygosity. Furthermore, significant MLH-growth relationships or single-locus heterozygosity-growth relationships within crosses must persist and be stronger after the effects of interfamily variation are removed (David, 1998). Rejection of the allozyme-associated heterosis by local true/associative overdominance effects would support the general dominance hypothesis. This would indicate that allozyme loci are generally not important predictors of heterosis in Eisenia.

\section{Materials and methods}

Eisenia fetida and E. andrei are popular commercial earthworms and their biology is relatively well known. They were first described as different morphotypes of Eisenia fetida according to differences in body pigmentation (André, 1963). Many authors now accept Eisenia fetida and Eisenia andrei as different species, although most older literature refers to these species collectively as Eisenia fetida. As separate species E. fetida corresponds to the striped morph, whereas $E$. andrei corresponds to the uniformly reddish morph. Aside from the differences in pigmentation, the two species are morphologically similar (Reinecke \& Viljoen, 1991). Fixed allelic differences exist at the mannose phosphate isomerase (Mpi) (Henry, 1999), the phosphoglucomutase $(P g m)$ loci, and the alanyl-amino peptidase (Aap) locus (Jaenike, 1982). Furthermore, E. fetida is polymorphic at the glucose phosphate isomerase (Gpi) locus, whereas E. andrei is monomorphic (Jaenike, 1982). The present results support the separate species designation.

Cocoon production by earthworms occurs continuously throughout the year provided environmental conditions are favourable (Edwards \& Bohlen, 1996; Presley et al., 1996). Both species are hermaphrodites and they mostly reproduce by outcrossing (Hartenstein et al., 1979). Presley et al. (1996) showed that Eisenia andrei did not produce viable cocoons by selfing. Likewise Eisenia fetida did not produce viable cocoons by selfing (pers. obs.), although selfing could occur rarely in both species.

Previous studies on the earthworm E. andrei suggest that early growth is related significantly to age of maturity and fecundity of individuals (Presley et al., 
1996). Therefore, early growth is an important physiological measure and a good predictor of organismal fitness in E. andrei. Significant allozyme heterosis has been detected for growth in $E$. andrei under conditions of environmental stress (low food, low soil moisture) for some polymorphic loci (Audo \& Diehl, 1995). The loci that showed a significant correlation encoded enzymes that were related to the glycolytic pathway, which was likely affected by these environmental stressors (Williams \& Diehl, 1992). Other studies have shown no relationship between heterozygosity and growth in E. andrei under similar environmental conditions (Diehl, 1988; McElroy et al., 1997).

Four stock populations of earthworms (two E.fetida and two E. andrei) were purchased from the following companies; Nasco (E. fetida-1), L.S.F. (E. fetida-2), Starkville Icehouse (E. andrei-1), and Henry Fields (E. andrei-2). The earthworm population purchased from Nasco was modified to remove apparent $E$. andrei individuals from the population. The populations have been maintained in the laboratory at Mississippi State University in the Department of Biological Sciences since June 1995. Previous population histories are unknown. The estimated adult population size for each culture was: E.f.-1, 1475; E.f.-2, 2350; E.a.-1, 11000 ; E.a.-2, 2400.

In June 1997 individual juvenile earthworms (aclitellate, $20.0 \mathrm{mg}$ ) were selected haphazardly from each of the four stock populations and raised in peat moss in $10 \mathrm{~mL}$ plastic vials (800 vials total: 200 earthworms/ population; $n=800$ earthworms). Temperature was maintained at $20^{\circ} \mathrm{C}$ in a Freas 818 Precision Scientific incubator and moisture was maintained at $4.0 \mathrm{~mL} \mathrm{~g}^{-1}$ dry peat moss. Commercial earthworm food (Magic Products, Inc., Amherst junction, WI) mixed with soyprotein baby formula was provided to the earthworms ad libitum. A pH of 7.0 was maintained in the peat moss by the addition of $\mathrm{CaCO}_{3}(5 \% \mathrm{w} / \mathrm{w})$. The earthworms were raised until sexual maturity occurred, as indicated by the development of the clitellum (at about 12 weeks age).

Fresh weight of individual earthworms in each generation was measured and recorded once every two weeks for 12 weeks. Earthworms were removed from the vial, rinsed in aged tap water and blotted dry. After weighing, the individuals were returned to their respective vials with fresh peat moss and food. Individuals found dead were placed in microcentrifuge tubes and frozen at $-80^{\circ} \mathrm{C}$ for genetic analysis.

Crosses were made between all possible combinations of the four experimental populations (total number of population crosses $=10$; Table 1). Mating partners were assigned haphazardly based on the individual identification numbers (25 parent pairs/cross; total number of
Table 1 Experimental crosses and sample sizes for two populations each of Eisenia fetida (E.f.-1 and -2) and $E$. andrei (E.a.-1 and -2) . The first number represents the number of offspring collected from each cross. The numbers in parentheses represent the number of families represented in each cross

\begin{tabular}{lcccc}
\hline & E.f.-1 & E.f.-2 & E.a.-1 & E.a.-2 \\
\hline E.f.-1 & $118(14)$ & $48(7)$ & $0(0)$ & $0(0)$ \\
E.f.-2 & $* * *$ & $21(3)$ & $0(0)$ & $0(0)$ \\
E.a.-1 & $* * *$ & $* * *$ & $175(19)$ & $138(17)$ \\
E.a.-2 & $* * *$ & $* * *$ & $* * *$ & $155(19)$ \\
\hline
\end{tabular}

crosses $=250$ ). Parental individuals were weighed and placed into a $50-\mathrm{mL}$ plastic cup with a portion of their native soil and given additional fresh peat moss and food. The crosses were all conducted in the same environment experienced by the parental generation. The mating pairs were allowed to cohabitate for three days. After this period the earthworms were separated and placed individually into $100 \mathrm{~mL}$ plastic cups. The earthworms were monitored for 2 months to allow for cocoon production and development of hatchlings (Meyer \& Bouwman, 1997).

The breeding cups were checked weekly for cocoons and offspring. Offspring were collected from the parental breeding cups when they reached $20.0 \mathrm{mg}$ and placed in individual vials with fresh peat moss and food. The offspring were grown in the same experimental environment in which the parents were raised. For logistic purposes only the first five offspring from each parent (10 offspring per parent pair) were used for early growth measurements $(10$ earthworms/cross $\times 250$ crosses $=2500$ offspring; 250 offspring/cross). The parents were allowed to produce cocoons for two months after which they were collected and frozen at $-80^{\circ} \mathrm{C}$ for genetic analysis. The two-month period was used to minimize mortality in the parental population and loss of genetic data.

Allozyme data were collected for glycerol-3-phosphate dehydrogenase (Gpd2; EC 1.1.1.8), malate dehydrogenase ( $M d h$; EC 1.1.1.37), alanyl amino peptidase (Aap; EC 3.4.1.-), esterase 2 (Est2; EC 3.1.1.-), phosphogluco-mutase (Pgm; EC 5.4.2.2), mannose phosphate isomerase (Mpi; EC 5.3.1.8), glucose-phosphate isomerase (Gpi; EC 5.3.1.9) and 6-phosphogluconate dehydrogenase ( $P g d$; EC 1.1.1.44). Polymorphism at these loci was detected using horizontal starch-gel electrophoresis. The patterns of inheritance of these allozymes conformed to Mendelian expectations.

Tissues were prepared according to Diehl (1988). The posterior end (postclitellar region) was used because of apparent protease activity in the anterior portion (Diehl, 
1988). The earthworm tissue was homogenized in $100 \mu \mathrm{L}$ of grinding buffer $(0.05 \mathrm{~m}$ Tris $/ \mathrm{HCl}, \mathrm{pH} 8.0$ with $20 \%$ glycerol and $0.1 \% \beta$-mercaptoethanol). The homogenate was sonicated to burst the cells and centrifuged at $10000 \mathrm{~g}$ for $15 \mathrm{~min}$. The supernatant was the source of the enzymes.

The gene products of Gpd2, Mdh and Aap were resolved with an amine-citrate buffer (Clayton \& Tretiak, 1972). The gene products of Est2, Pgm, Mpi, Gpi and $P g d$ were resolved with a Tris-maleate buffer (Selander et al., 1971). Enzymes were stained according to Diehl (1988) and Garton et al. (1984). The common alleles were recorded as the 100 allele. Other alleles at each locus were recorded as the 110 allele or the 90 allele depending on the electrophoretic mobility with respect to the common allele. The common alleles have been determined from the previous work of Diehl (1988). Mpi was used as a diagnostic locus to identify every individual earthworm as either Eisenia fetida or E. andrei.

The morphometric and genetic data were analysed in accord with the hypotheses stated above. A heterosis value for growth rate of each individual offspring compared to that of the better parent's growth rate was calculated from the equation ( $\mathrm{Li} \& \mathrm{Wu}, 1996)$ :

$\left[\left(F_{1}\right.\right.$ value High-parent value $) /$

(High-parent value) $] \times 100$.

This calculation generated a traditional heterosis value that could be used to compare the average heterosis among populations. Growth rate was calculated as a direct measure of change in weight over time. The heritable genetic variation for growth rate was estimated from the simple linear regressions of offspring growth rate values and mid-parent growth rate values for all crosses. Tests for conformity to Hardy-Weinberg equilibrium, heterozygote deficiencies and excesses, and mean observed heterozygosity per locus were calculated using BIOsYs (Swofford \& Selander, 1989, release 1.7) and GENEPOP (Raymond \& Rousset, 2000; v.3.2). Relationships between individual multilocus allozyme heterozygosity (MLH) and growth rate were calculated with simple linear regression for all parent and offspring populations.

The hierarchical experimental design was used to distinguish the local from general effects models of heterosis. If significant correlations between MLH and growth rate are detected at the within-family level where the effects of inbreeding are presumed to be homogeneous, then the correlation between multilocus allozyme heterozygosity and growth rate is not the result of general effects of between family variance in the level of inbreeding. In order to eliminate effects owing to between-family variance for growth rate and evaluate whether the correlation persisted, standard deviates from family means were used (Bierne et al., 1998) All statistical analyses were performed with programs of SAS (SAS Institute, Inc., Cary, NC) and systat (SPSS Inc. 1997, v.7.0).

\section{Results}

Nei's (1978) genetic distance calculations based on allozyme frequencies within each population suggest that Eisenia fetida and $E$. andrei are genetically distinct species (Table 2). Within each species the populations are genetically similar (Table 2). The fact that no interspecific hybrids were produced in this experiment compromised the power of the experimental design in distinguishing among the possible mechanisms of heterosis (Table 1). In retrospect, it would have been useful to analyse more individuals per cross and reduce the number of crosses. These data support the recent species designation of Eisenia fetida and E. andrei. Thus, intraspecific variation was examined before proceeding to comparisons between the species. Patterns of allozyme heterozygosity and heterosis were compared between populations within each species and between the two species.

\section{Eisenia fetida}

The parent populations were in Hardy-Weinberg equilibrium for all loci with the exception of Est2 in population E.f.-1. This locus also had significantly less heterozygosity than expected $(\mathrm{D}=-0.512)$ (Tables 3 and 4). Population E.f.-1 was polymorphic at $62.5 \%$ of the eight loci surveyed and had a mean observed heterozygosity per locus $(H)$ of 0.266 . Population E.f.-2 was polymorphic at $75.0 \%$ of the eight loci surveyed with $H=0.271$ (Table 5).

The offspring populations of E. fetida were in Hardy-Weinberg equilibrium for most loci (Table 3).

Table 2 Nei (1978) unbiased genetic identity (below the diagonal) and Nei (1978) unbiased genetic distance (above the diagonal) among the four parent populations of Eisenia fetida and E. andrei (see caption to Fig. 1)

\begin{tabular}{lcccc}
\hline & E.f.-1 & E.f.-2 & E.a.-1 & E.a.-2 \\
\hline E.f.-1 & $* * * *$ & 0.005 & 0.465 & 0.528 \\
E.f.-2 & 0.995 & $* * * *$ & 0.487 & 0.571 \\
E.a.-1 & 0.628 & 0.614 & $* * * *$ & 0.031 \\
E.a.-2 & 0.590 & 0.565 & 0.969 & $* * * *$ \\
\hline
\end{tabular}




\begin{tabular}{lcccccc}
\hline & \multicolumn{2}{c}{ Parent populations } & & \multicolumn{3}{c}{$\mathrm{F}_{1}$ populations } \\
\cline { 2 - 3 } \cline { 5 - 7 } Locus & E.f.-1 & E. f.-2 & & E.f.- $1 \times$ E.f.-1 & E.f.- $2 \times$ E.f.-2 & E.f.-1 $\times$ E.f.-2 \\
\hline$M p i$ & - & - & & - & - & - \\
$P g m$ & 0.143 & 1.000 & & 0.168 & $0.021^{*}$ & 0.112 \\
Pgd & 1.000 & 0.248 & & 0.086 & 1.000 & 1.000 \\
Est 2 & $0.005^{* *}$ & 0.099 & & 0.069 & 1.000 & 0.260 \\
Aap & 1.000 & 0.183 & & 1.000 & $0.025^{*}$ & 1.000 \\
$M d h$ & - & - & & - & - & - \\
$G p d 2$ & 0.733 & 0.593 & & 0.240 & 0.394 & 0.098 \\
$G p i$ & - & 0.373 & - & 0.540 & 1.000 \\
\hline$* P<0.05,{ }^{* *} P<0.01$. & & & & &
\end{tabular}

\begin{tabular}{|c|c|c|c|c|c|}
\hline \multirow[b]{2}{*}{ Locus } & \multicolumn{2}{|c|}{ Parent populations } & \multicolumn{3}{|c|}{$F_{1}$ populations } \\
\hline & E.f.-1 & E.f.-2 & E.f.- $1 \times$ E.f. -1 & E.f. $-2 \times$ E.f. -2 & E.f. $-1 \times$ E.f.- 2 \\
\hline$M p i$ & - & - & - & - & - \\
\hline$P g m$ & $0.446^{*}$ & 0.121 & -0.039 & -0.383 & $0.478^{*}$ \\
\hline$P g d$ & 0.080 & -0.425 & -0.173 & 0.108 & 0.064 \\
\hline Est2 & $-0.512 * *$ & -0.517 & $-0.203^{*}$ & -0.096 & -0.193 \\
\hline Aap & 0.016 & -0.420 & 0.019 & $-0.531^{*}$ & 0.016 \\
\hline$M d h$ & - & - & - & - & - \\
\hline Gpd2 & -0.069 & 0.193 & 0.128 & 0.220 & $0.297 *$ \\
\hline Gpi & - & -0.274 & - & -0.231 & 0.122 \\
\hline Average & -0.008 & -0.179 & -0.046 & -0.152 & 0.131 \\
\hline
\end{tabular}

$* P<0.05,{ }^{* *} P<0.01$.

\begin{tabular}{|c|c|c|c|c|c|}
\hline \multirow[b]{2}{*}{ (a) } & \multicolumn{2}{|c|}{ Parent populations } & \multicolumn{3}{|c|}{$\mathrm{F}_{1}$ populations } \\
\hline & E.f.-1 & E.f.-2 & E.f.- $1 \times$ E.f.- 1 & E.f. $-2 \times$ E.f. -2 & E.f. $-1 \times$ E.f.- 2 \\
\hline$P(\%)$ & 62.5 & 75.0 & 62.5 & 75.0 & 75.0 \\
\hline$H$ & 0.266 & 0.271 & 0.216 & 0.254 & 0.318 \\
\hline SE & $(0.123)$ & $(0.086)$ & $(0.085)$ & $(0.063)$ & $(0.112)$ \\
\hline (b) & E.a.-1 & E.a.-2 & E.a. $-1 \times$ E.a. -1 & E.a. $-2 \times$ E.a. -2 & E.a. $-1 \times$ E.a. -2 \\
\hline$P(\%)$ & 87.5 & 87.5 & 87.5 & 87.5 & 87.5 \\
\hline$H$ & 0.408 & 0.361 & 0.368 & 0.329 & 0.460 \\
\hline SE & $(0.070)$ & $(0.062)$ & $(0.064)$ & (0.059) & $(0.075)$ \\
\hline
\end{tabular}

Table 3 Hardy-Weinberg expectation significance test using exact probabilities for all loci surveyed in the parent and $F_{1}$ populations of Eisenia fetida. The loci Mpi and Mdh were monomorphic in the Eisenia fetida populations surveyed
Table 4 Heterozygote deficiencies $(D)$ for all loci surveyed in the parent and $\mathrm{F}_{1}$ populations of Eisenia fetida
Table 5 Percentage of polymorphic loci $(P)$ and the mean observed heterozygosity per locus $(H)$ of the polymorphic loci and standard error (SE) for all loci surveyed in the parent and $F_{1}$ populations of (a) Eisenia fetida and (b) E. andrei
Significant heterozygote deficiencies were detected in the intrapopulation crosses, and significant heterozygote excesses were detected in the interpopulation crosses (Table 4). The percentage of polymorphic loci in the $F_{1}$ populations was the same as that of the parent populations. The value of $H$ in the intrapopulation crosses was less than the calculated value in the corresponding parent values but was not significantly different ( $H=0.216$ and 0.254 for E.f.- 1 and E.f.-2, respectively).
The value of $H$ for the interpopulation crosses was greater than that of either parent population $(H=0.318)$. The value of $H$ for the offspring was not significantly greater than that of the parent population that had the greatest mean observed heterozygosity per locus (Table 5).

Growth rates differed between parent populations of E. fetida (Fig. 1; $P<0.001$ ); however, differences in growth rate observed between the parent populations 
Fig. 1 The average growth rates (plus SE) among the four parent populations of Eisenia fetida (E.f.-1 and -2) and E. andrei (E.a.-1 and -2). Comparisons were made between populations within and between species.

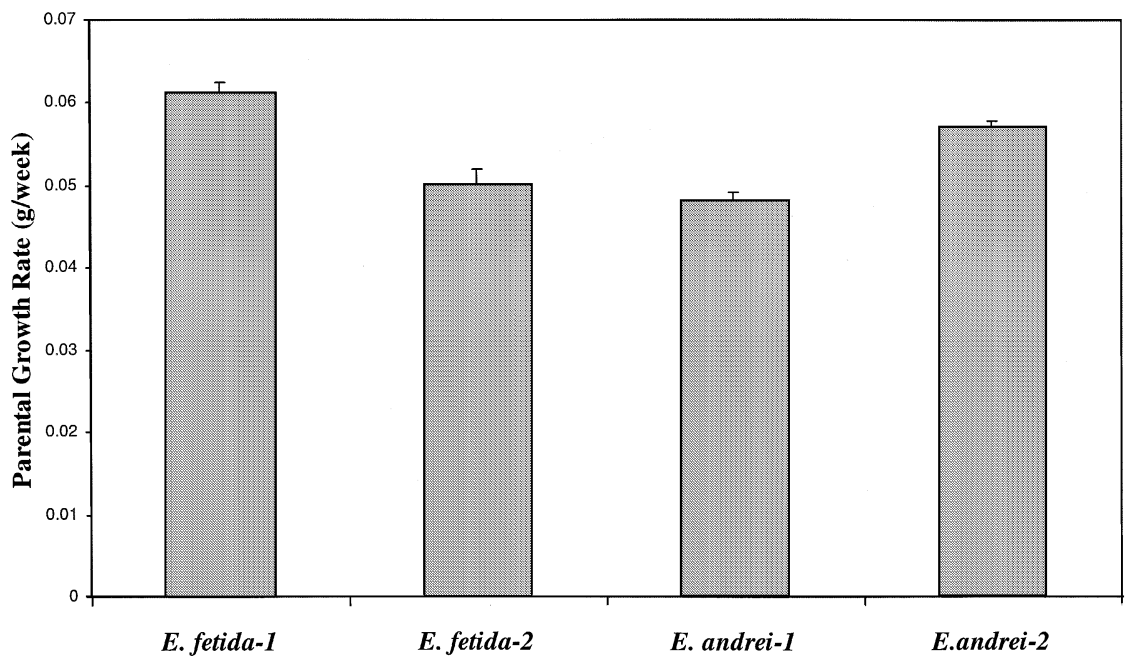

were not observed in the $F_{1}$ generation of the intrapopulation crosses. Offspring populations of E. fetida had significantly greater average growth rate than parent populations in all crosses except the E.f.- $1 \times$ E.f.- 1 $(P<0.01$; Fig. 2). Within the offspring of E. fetida, there were no differences in growth rate among the crosses (Fig. 2).

Heterosis for growth rate (performance of the offspring compared to that of the better growing parent) detected in the E. fetida interpopulation cross was intermediate to that of the intrapopulation crosses indicating that there was not a strong relationship between population heterozygosity and heterosis (Fig. 3). Growth rate of the offspring was not related to the growth rate of the parents in any of the E. fetida crosses indicating a lack of significant heritable genetic variation for growth rate in these crosses. Multilocus heterozygosity (MLH) and growth rate were not correlated in any of the crosses or parent populations.

\section{Eisenia andrei}

The parent populations were in Hardy-Weinberg equilibrium for all loci with the exception of $P g d$ in the E.a.-1 population. This locus also had significantly less heterozygosity than expected $(D=-0.458)$ (Tables 6 and 7). Both populations of Eisenia andrei were polymorphic at $87.5 \%$ of the loci. Population E.a.-1 had a mean observed heterozygosity per locus $(H)$ of 0.408 ; $H=0.361$ for E.a.-2 (Table 5).

The offspring populations of E. andrei were in HardyWeinberg equilibrium for most loci (Table 6). Significant heterozygote deficiencies were detected in the intrapopulation crosses, and significant heterozygote excesses were detected in the interpopulation crosses (Table 7). The percentage of polymorphic loci in the $F_{1}$ populations was the same as that of the parent populations. The values for $H$ in the intrapopulation crosses were less than the calculated value in the corresponding
Fig. 2 The average growth rates (plus SE) of the parents within a cross and the offspring of the four parent populations of Eisenia fetida (E.f.-1 and -2) and E. andrei (E.a.-1 and -2). The average growth rate of the parents is represented by the left bar with the bar on the right with diagonal striping representing the growth rates for the $\mathrm{F}_{1}$.

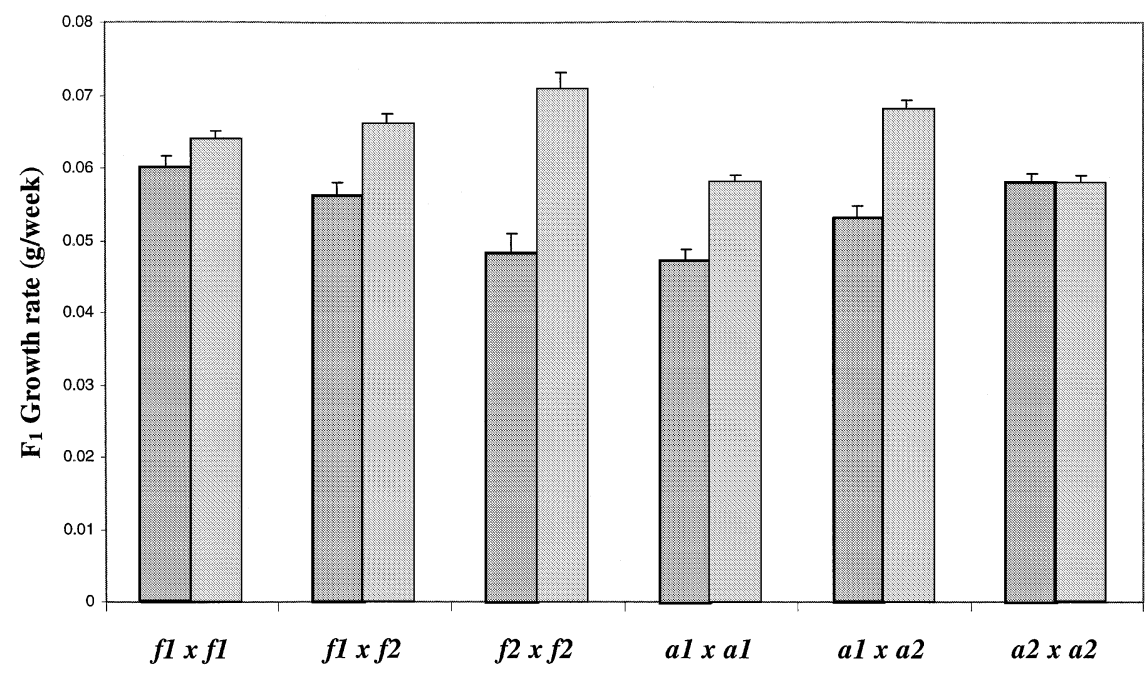

(C) The Genetics Society of Great Britain, Heredity, 87, 598-608. 


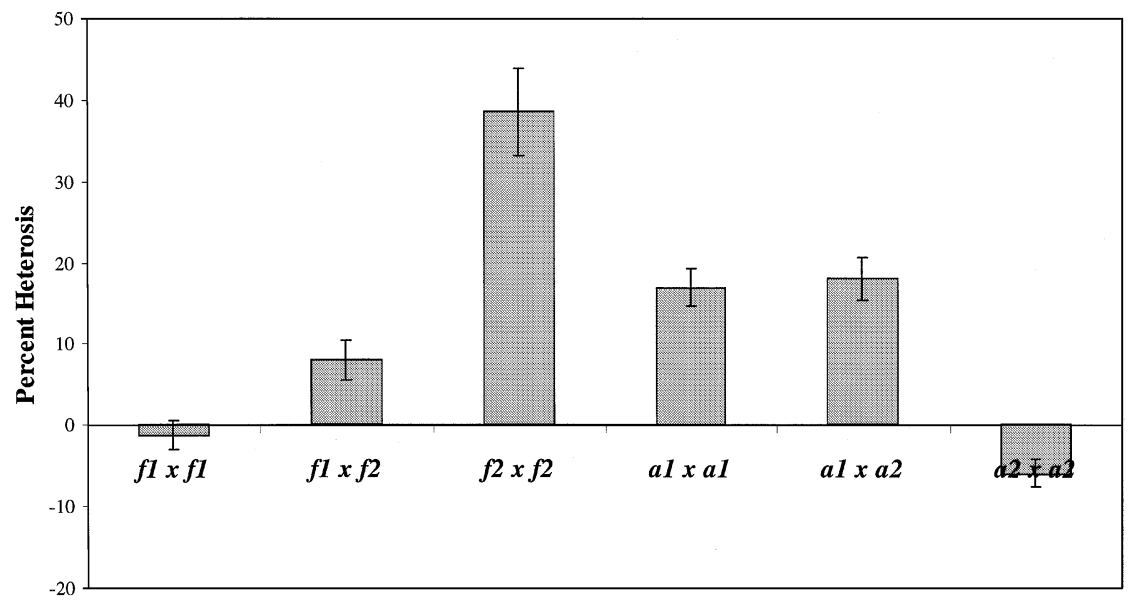

Fig. 3 Heterosis in the $F_{1}$ generation $\left(\%\right.$ Heterosis $=\left[\left(\mathrm{F}_{1}\right.\right.$ growth rate - high parent growth rate)/high parent growth rate]* (100). parent values but was not significantly different ( $H=0.368$ and 0.329 for E.a.-1 and E.a.-2, respectively). The value for $H$ for the interpopulation crosses was greater than that of either parent population $(H=0.460)$; however, $H$ for the offspring was not significantly greater than that of the parent population that had the greatest mean observed heterozygosity per locus (Table 5).

Growth rates differed among parent populations of E. andrei (Fig. 1; $P<0.001$ ); however, differences in growth rate observed between the parent populations were not observed in the $F_{1}$ generation. Offspring populations of $E$. andrei had significantly greater

\begin{tabular}{lcccccc}
\hline & \multicolumn{2}{c}{ Parent populations } & \multicolumn{3}{c}{$\mathrm{F}_{1}$ populations } \\
\cline { 2 - 3 } \cline { 5 - 6 } Locus & E.a.-1 & E. a.-2 & & E.a.- $1 \times$ E.a.-1 & E.a. $-2 \times$ E.a.-2 & E.a.-1 $\times$ E.a.-2 \\
\hline$M p i$ & 1.000 & 1.000 & & 0.412 & $0.017^{*}$ & $0.010^{* *}$ \\
Pgm & 1.000 & 1.000 & & 1.000 & 0.090 & 0.389 \\
Pgd & $0.006^{* *}$ & 1.000 & & $0.000^{* * *}$ & 0.291 & 0.162 \\
Est2 & 0.207 & 0.752 & & $0.043^{*}$ & $0.004^{* *}$ & 0.625 \\
Aap & 1.000 & 0.501 & & 0.739 & 0.091 & $0.050^{*}$ \\
$M d h$ & 1.000 & 0.763 & & 0.725 & 0.630 & $0.003^{* *}$ \\
$G p d 2$ & 1.000 & 0.546 & 1.000 & 0.865 & 0.804 \\
$G p i$ & - & - & - & - & - \\
\hline${ }^{*} P<0.05,{ }^{* *} P<0.01,{ }^{* * *} P<0.001$. & - & & -
\end{tabular}

\begin{tabular}{lcrcccc}
\hline & \multicolumn{2}{c}{ Parent populations } & & \multicolumn{3}{c}{$\mathrm{F}_{1}$ populations } \\
\cline { 2 - 3 } \cline { 5 - 7 } Locus & E.a.-1 & E. a.-2 & & E.a.-1 $\times$ E.a.-1 & E.a.-2 $\times$ E.a.-2 & E.a.-1 $\times$ E.a.-2 \\
\hline$M p i$ & 0.038 & 0.096 & & -0.071 & $-0.211^{* *}$ & $0.326^{* *}$ \\
Pgm & 0.021 & -0.018 & & 0.005 & -0.138 & -0.122 \\
Pgd & $-0.458^{* *}$ & 0.025 & & $-0.606^{* *}$ & -0.103 & 0.208 \\
Est2 & 0.212 & 0.082 & & $-0.178^{*}$ & $-0.241^{* *}$ & 0.081 \\
Aap & 0.038 & 0.117 & & 0.030 & -0.148 & $0.242^{*}$ \\
Mdh & -0.012 & 0.075 & & -0.034 & 0.045 & $0.363^{* *}$ \\
Gpd2 & -0.156 & 0.097 & & -0.004 & -0.024 & 0.052 \\
Gpi & - & - & & - & - & - \\
Average & -0.045 & 0.068 & $-0.123^{*}$ & $-0.117^{*}$ & $0.164^{*}$ \\
\hline
\end{tabular}

$* P<0.05, * * P<0.01$.
Table 6 Hardy-Weinberg expectation significance test using exact probabilities for all loci surveyed in the parent and $F_{1}$ populations of Eisenia andrei. The locus Gpi was monomorphic in the E. andrei populations surveyed
Table 7 Heterozygote deficiencies $(D)$ for all loci surveyed in the parent an $\mathrm{F}_{1}$ populations of Eisenia andrei 
Fig. 4 The heritability of growth rate within the E.a. $-1 \times$ E.a.-1 cross. Heritability was calculated based on the regression of offspring growth rate on the mid-parent value of each family (growth rate $=0.375 x+0.040 ; r^{2}=0.065$; $P<0.001 ; N=149)$.

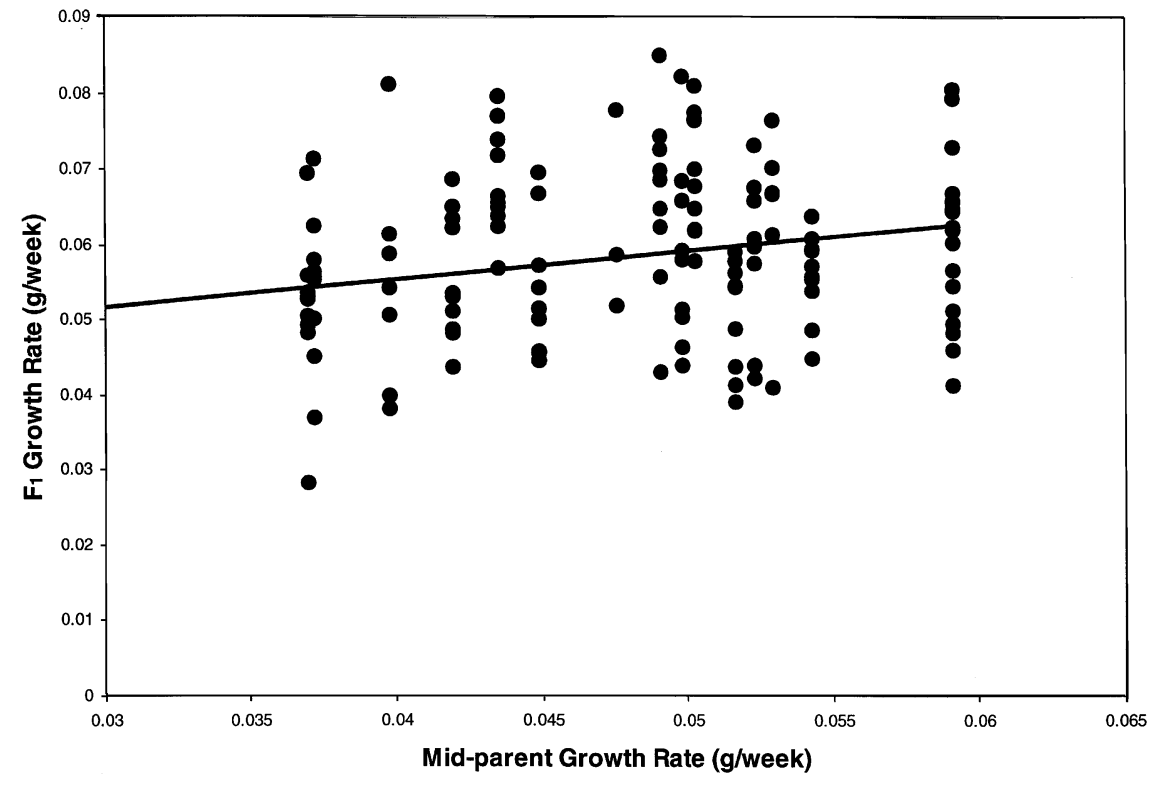

average growth rate than parent populations in all crosses except the E.a. $-2 \times$ E.a.-2 $(P<0.01$; Fig. 2). Within the offspring of $E$. andrei, growth rate was greatest in the interpopulation cross (Fig. 2).

Heterosis for growth rate detected in the $E$. andrei interpopulation cross was greater than either of the intrapopulation crosses (Fig. 3), indicating that there was a positive relationship between increased population heterozygosity and the average heterosis for growth rate of individuals in that population. There was a significant, positive relationship between the mid-parent value for growth rate and the growth rate of the offspring in the E.a.- $1 \times$ E.a.-1 cross indicating the presence of significant heritable variation for growth rate (Fig. $4 ; P<0.001, h^{2}=0.375$ ). There was a significant, positive relationship between multilocus heterozygosity $(\mathrm{MLH})$ and growth rate in the E.a.-1 $\times$ E.a.-1 $\mathrm{F}_{1}$ population (Fig. $5 ; r^{2}=0.039 ; P<0.02$ ). However heterosis for growth rate was not correlated with individual multilocus heterozygosity in any of the parent populations or other crosses.

The hierarchical experimental design was used to distinguish the local from general effects models of heterosis. Overall, the effect of allozyme genotype was not consistent among any of the population crosses or even among generations within a species or a population. A large portion of the variance in growth rate and heterosis was between-family variance. Individuals within a family did not differ with respect to growth rate or heterosis (model; $y=$ species $+\operatorname{species(cross)~}+$ species[cross(family)]). In order to eliminate effects owing to between-family variance for growth rate within each cross and evaluate whether the MLH-growth rate correlation was detected or persisted, standard deviates from family means were used (Bierne et al., 1998). There was not a significant, positive relationship between multilocus heterozygosity and the standard deviates from the family mean detected in the E.a. $-1 \times$ E.a. $-1 F_{1}$ population (Fig. $6 ; r^{2}=0.013 ; P=0.162$ ). Thus, the significant relationship between multilocus heterozygosity and growth detected in the E.a. $-1 \times$ E.a. $-1 F_{1}$ population was likely the result of general dominance effects and not local true/associative overdominance effects.

\section{Discussion}

The importance of heterosis in the evolution of natural populations has been debated for almost eight decades but no consensus has been reached. Analysis of detailed data on specific allozyme genotypes will be an integral part of advancement towards understanding the role of allozyme variation in natural populations. This study was important for determining the effect of specific allozyme loci on heterosis. The results of this study provide (i) data both to support a positive relationship between heterozygosity and fitness for one species and reject it for the other species and (ii) data to support the general dominance effects hypothesis for allozymeassociated heterosis.

The observed patterns of heterosis included two anticipated forms. A significant positive relationship between individual allozyme heterozygosity and growth rate and a concurrent increase in population heterozygosity and growth rate. However, these observations were not consistent among populations or crosses. 

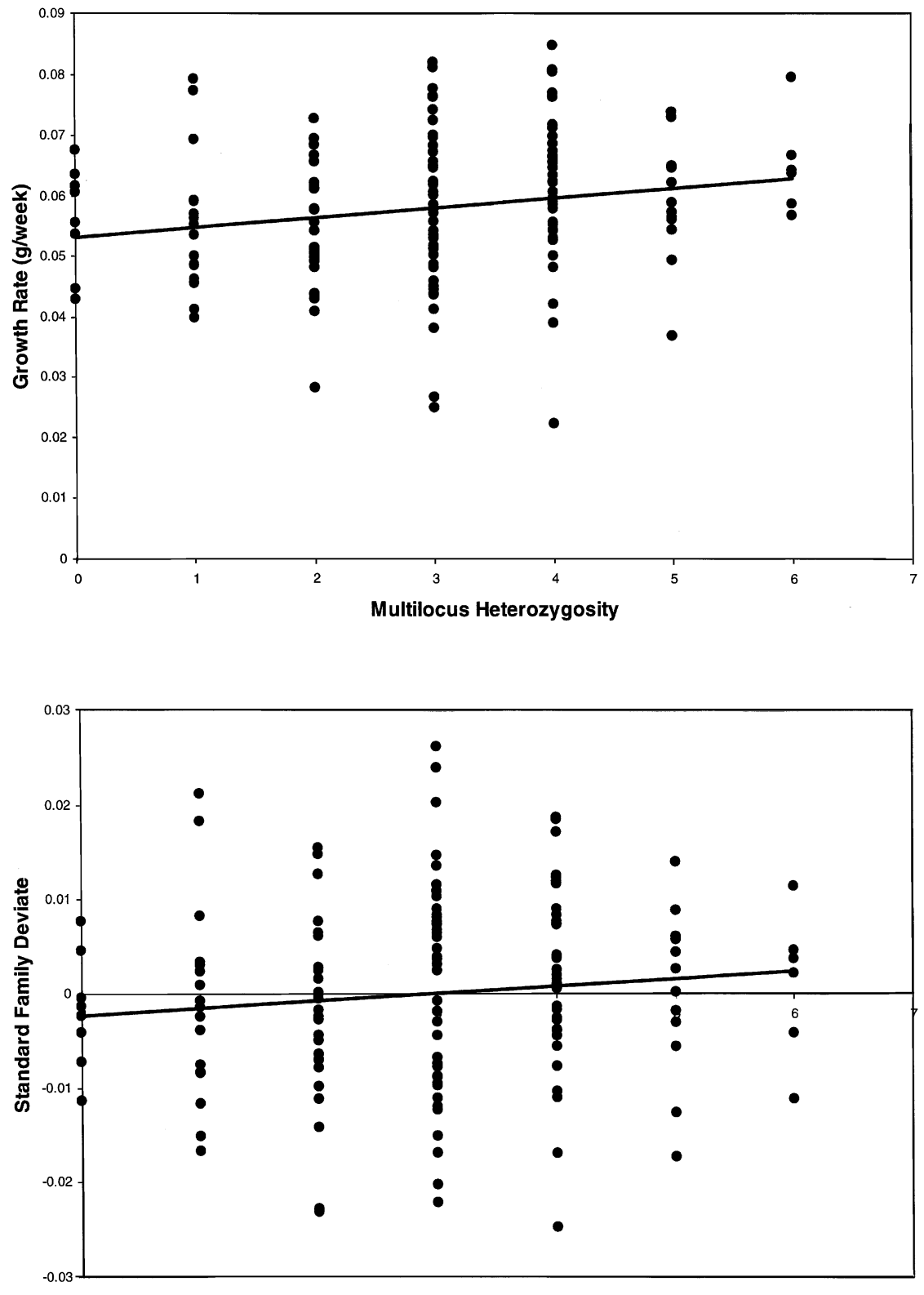

Multilocus Heterozygosity
Fig. 5 The relationship of multilocus heterozygosity (MLH) and growth rate within the E.a. $-1 \times$ E.a. -1 cross $\left(P<0.02 ; r^{2}=0.039 ; n=149\right)$.
Fig. 6 The relationship of multilocus heterozygosity (MLH) and standard deviates from family mean within the E.a. $-1 \times$ E.a. -1 cross $(P=0.162$; $\left.r^{2}=0.013 ; n=149\right)$.
Heterosis was also detected in crosses where there was no significant positive relationship between individual allozyme heterozygosity and growth rate or a concurrent increase in population heterozygosity and growth rate.

Growth rate differed between parent populations within a species, but the average growth rate between species was not different. All of the parent populations were raised in the same environmental conditions. The observation of differences in growth rate between populations of the same Eisenia species grown under similar environmental conditions is not without precedent (Reineke \& Viljoen, 1991). Differences in growth rate between species may be dependent on the growth medium (Elvira et al., 1996) or the stock population used in the studies (Reineke \& Viljoen, 1991). The population sizes were large enough that inbreeding and large effects of genetic drift should not have been significant factors; however, the exact long-term history of these populations is unknown.

If the growth trajectory is dependent on environmental factors early in ontogeny, it is possible that the differences in growth rate between the parent populations within a species were caused by environmental differences present in the stock population environment despite efforts to create homogeneous conditions among 
the stock housings. The environment provided for the earthworms in both generations has been shown to produce maximal fitness with respect to growth, reproduction and survivorship (Presley et al., 1996). If maximal growth rate was limited by environmental factors experienced early in ontogeny by the parental stock populations, then the observed increase in growth rate in the intrapopulation crosses from the parents that had the least growth within each species was the result of a release from the environmental constraints on growth rate in those populations. The observed increase in growth rate in the interpopulation crosses could be the result of both a release from the environmental constraints on growth rate and unique genetic combinations present in those populations.

The inconsistency of allozyme genotype effects detected among all of the experimental populations suggests that genetic background within populations is an important factor. Thus the allozyme markers themselves are not likely responsible for the observed heterosis in these populations; rather, they are marking chromosomal segments that may contain deleterious recessive mutations in the genome or linked loci that are the targets of selection in E. andrei. There are many mechanisms that may cause heterosis. The problem with allozyme-associated heterosis and heterosis in general has been the predictability of the phenomenon. The present data indicate that simple heritability of a fitness-related trait may be necessary for predictive power and repeatability of allozymeassociated heterosis. When variation for growth rate was heritable there was a significant, positive relationship between the multilocus heterozygosity (MLH) of an individual and growth rate. Otherwise the magnitude of heterosis was unpredictable at both the species and population levels. Thus, allozyme-associated heterosis and heterosis for growth rate are not tightly coupled phenomena.

The significant allozyme-associated heterosis detected in the E.a. $-1 \times$ E.a.-1 intrapopulation cross was coupled with significant additive genetic variation for growth rate. Furthermore, the significant, positive relationship between MLH and growth rate did not persist after the effects of between family variance were taken into account (Figs 5 and 6). These data empirically tested the prediction that local genetic effects, which may cause allozyme-associated heterosis correlations, should appear stronger in homogeneous genetic backgrounds (David, 1998). These data, coupled with a general lack of a significant, positive relationship between MLH and growth rate in other crosses with similar breeding histories in this experiment, support the general overdominance effects hypothesis for growth rate heterosis in E. andrei.

\section{Acknowledgements}

We thank Melinda Chow, Benji Makamson and Ann Gutendorf for assistance in data collection and earthworm husbandry. We also thank Joel Trexler, John Brookfield and two anonymous reviewers for helpful comments on this manuscript. This research was supported by NSF grant DEB-9221094 to WJD and a Sigma Xi Grant-in-Aid of Research to TCM.

\section{References}

ANDRÉ, F. 1963. Contribution a l'analyse experimental de la reproduction des lombriciens. Bull. Biol. Fr. Belg., 97, $1-101$.

AUDO, M. C. AND DIEHL, w. J. 1995. Effect of quantity and quality of environmental stress on multi locus heterozygosity-growth relationships in Eisenia fetida. Heredity, 75, 98-105.

BIERNE, N., LAUNEY, S., NACIRI-GRAVEN, Y. AND BONHOMME, F. 1998. Early effect of inbreeding as revealed by microsatellite analyses on Ostrea edulis larvae. Genetics, 148, 1893-1906.

BOOTH, C. L., WOODRUFF, D. S. AND GOULD, S. J. 1990. Lack of significant associations between allozyme heterozygosity and phenotypic traits in the land snail Cerion. Evolution, 44, 210-213.

BRITTEN, H. B. 1996. Meta-analysis of the association between multilocus heterozygosity and fitness. Evolution, 50, 2158-2164.

BRUCE, A. B. 1910. The Mendelian theory of heredity and the augmentation of vigor. Science, 32, 627-628.

CLAYTON, J. W. AND TRETIAK, D. N. 1972. Amine-citrate buffers for $\mathrm{pH}$ control in starch gel electrophoresis. J. Fish. Res. Board. Can., 29, 1169-1172.

CROw, J. F. 1952. Dominance and Overdominance. In: Gowen, J. W. (ed.) Heterosis: a Record of Researches Directed Toward Explaining and Utilizing the Vigor of Hybrids, pp. 282-297. Iowa State College Press, Ames, IA.

DAVID, P. 1998. Heterozygosity-fitness correlations: new perspectives on old problems. Heredity, 80, 531-537.

DIEHL, w. J. 1988. Genetics of carbohydrate metabolism and growth in Eisenia foetida (Oligochaeta: Lumbricidae). Heredity, 61, 379-387.

EAST, E. M. 1936. Heterosis. Genetics, 21, 375-397.

EDWARDS, C. A. AND BOHLEN, P. J. 1996. Biology and Ecology of Earthworms, 3rd edn. Sinauer Associates, Sunderland, MA.

ELVIRA, C., DOMINEQUEZ, J. AND BRIONES, M. J. I. 1996. Growth and reproduction of Eisenia andrei and E. fetida (Oligochaeta, Lumbricidae) in different organic residues. Pedobiologia, 40, 377-384.

GARTON, D. W., KOEHN, R. K. AND SCOTT, T. M. 1984. Multiplelocus heterozygosity and the physiological energetics of growth in the coot clam, Mulinia lateralis, from a natural population. Genetics, 108, 445-455.

HARTENSTEIN, R., NEUHAUSER, E. F. AND KAPLAN, D. L. 1979. Reproductive potential of the earthworm Eisenia fetida. Oecologia, 43, 329-340. 
HARTL, D. L. AND ClARK, A. G. 1989. Principles of Population Genetics. Sinauer Associates, Sunderland, MA.

HAYES, H. K. 1952. Development of the heterosis concept. In: Gowen, J. W. (ed.) Heterosis: a Record of Researches Directed Toward Explaining and Utilizing the Vigor of Hybrids, pp. 49-65. Iowa State College Press, Ames, IA.

HENRY, W. B. 1999. Differentiation of allozyme loci to distinguish between two species of Eisenia. M.Sc. Thesis, Mississippi State University.

HOULE, D. 1989. The maintenance of polygenic variation in finite populations. Evolution, 43, 1767-1780.

JAENIKE, J. 1982. 'Eisenia foetida' is two biological species. Megadrilogica, 4, 6-8.

LI, B. AND WU, R. 1996. Genetic causes of heterosis in juvenile aspen: a quantitative comparison across intra- and interspecific hybrids. Theor. Appl. Genet., 93, 380-391.

LYNCH, M. AND WALSH, B. 1998. Genetics and Analysis of Quantitative Traits. Sinauer Associates, Sunderland, MA.

MCANDREW, B. J., WARD, R. D. AND BEARDMORE, J. A. 1986. Growth rate and heterozygosity in the plaice, Pleuronectes platessa. Heredity, 57, 171-180.

McElRoY, T. C., PRESLEY, M. L. AND DIEHL, W. J. 1997. Genotypes of multiple allozyme loci interact with an experimental environment to affect growth of juvenile earthworms (Eisenia fetida). Comp. Biochem. Physiol., 118B, 437-446.

McElRoY, T. C., PRESLEY, M. L. AND DIEHL, W. J. 1999. Genotypes of multiple allozyme loci interact with an experimental environment to affect survivorship in earthworms (Eisenia andrei). Comp. Biochem. Phys., 123A, 241-247.

MEYER, W. J. AND BOUWMAN, H. 1997. Anisopary in compost earthworm reproductive strategies (Oligochaeta). Soil Biol. Biochem., 29, 731-735.

mitton, J. B. 1997. Selection in Natural Populations. Oxford University Press, New York.

MUlVEY, M., Keller, G. P. AND MEFFe, G. K. 1994. Single- and multiple-locus genotypes and life-history responses of Gambusia holbrooki reared at two temperatures. Evolution, 48, 1810-1819.
NEI, M. 1978. Estimation of average heterozygosity and genetic distance from a small number of individuals. Genetics, $\mathbf{8 9}$, 583-590.

POGSON, G. H. AND FEVOLDEN, S. E. 1998. DNA heterozygosity and growth rate in the Atlantic cod Gadus morhua (L). Evolution, 52, 915-920.

POGSON, G. AND ZOUROS, E. 1994. Allozyme and RFLP heterozygosities as correlates of growth rate in the scallop Placopecten magellanicus: a test of the associative overdominance hypothesis. Genetics, 137, 221-231.

PRESLEY, M. L., MCELROY, T. C. AND DIEHL, W. J. 1996. Soil moisture and temperature interact to affect growth, survivorship, fecundity, and fitness in the earthworm Eisenia fetida. Comp. Biochem. Physiol., 114A, 319-326.

RAYMOND, M. AND ROUSSET, F. 2000. GENEPOP: Population Genetics Software for Exact Tests and Ecumenicism, v.3.2. Université de Montpellier II, France.

REINECKE, A. J. AND VILJoen, S. A. 1991. A comparison of the biology of Eisenia fetida and Eisenia andrei (Oligochaeta). Biol. Fert. Soils, 11, 295-300.

SELANDER, R. K., SMITH, M. H., YANG, S. Y., JOHNSON, W. E. AND GENTRY, J. B. 1971. Biochemical polymorphism and systematics in the genus Peromyscus. I. Variation in the old-field mouse (Peromyscus polionotus). Stud. Gen. VI. Univ. TX, 7103, 49-90.

SWOFFORD, D. L. AND SELANDER, R. B. 1989. Biosys-1. A computer program for the analysis of allelic variation in population genetics and biochemical systematics, Release 1.7. University of Illinois, Urbana, IL.

WHITLOCK, M. 1993. Lack of correlation between heterozygosity and fitness in forked fungus beetles. Heredity, 70, 574-581.

WILLIAMS, D. L. AND DIEHL, W. J. 1992. Interactive effects of soil moisture and food on glycolytic metabolism in Eisenia fetida (Oligochaeta). Comp. Biochem. Physiol., 102B, 911-917. 